\title{
Asymptotic structure of the attractor for processes on time-dependent spaces
}

\author{
Monica Conti, Vittorino Pata* \\ Politecnico di Milano - Dipartimento di Matematica "F. Brioschi", Via Bonardi 9, 20133 Milano, Italy
}

Received 16 August 2013

Accepted 11 February 2014

\section{Introduction}

This article is motivated by the asymptotic analysis of the semilinear wave equation on a bounded domain $\Omega \subset \mathbb{R}^{3}$

$$
\varepsilon u_{t t}+\alpha u_{t}-\Delta u+f(u)=g,
$$

where $f$ is a suitable nonlinearity and $g$ an external force. Here $\alpha$ is a strictly positive damping coefficient, while $\varepsilon=\varepsilon(t)$ is a positive function of $t$ fading to zero as time goes to infinity. Accordingly, we can formally interpret the parabolic equation

$$
\alpha u_{t}-\Delta u+f(u)=g
$$

as the limit situation in the longtime of (1.1). The model above, which can be seen as a nonlinear damped wave equation with time-dependent speed of propagation $1 / \varepsilon(t)$, has been studied in detail in [1]. In that work, problem (1.1) is shown to generate a process $U(t, \tau)$, acting on a family of time-dependent spaces $\left\{\mathcal{Z}_{t}\right\}$, possessing the time-dependent global attractor in the sense of [2]. Loosely speaking, this is the smallest family $\left\{A_{t}\right\}$ (where each $A_{t}$ lies in its own $t$-indexed space $Z_{t}$ ) which attracts bounded subsets in a pullback way. At the same time, Eq. (1.2) generates a semigroup $S(t)$ possessing the global attractor $A_{\infty}$, according to the classical definition [3-6]. It is then a natural question to investigate whether there is a closeness of $A_{t}$ to $A_{\infty}$ as $t \rightarrow \infty$. Indeed, although this is formally suggested by the very structure of the equations, $A_{\infty}$ is a forward attractor, that is, in principle, quite a different object from the pullback family $\left\{A_{t}\right\}$. Nonetheless, we may subsume the main result of the present paper by saying that

$$
A_{t} \rightarrow A_{\infty} \text { as } t \rightarrow \infty
$$

* Corresponding author. Tel.: +39 0223994583.

E-mail addresses: monica.conti@polimi.it (M. Conti), vittorino.pata@polimi.it (V. Pata). 
Of course, the type of convergence has to be properly defined, as we will see later. This somehow tells that (1.2) provides an accurate description of the longterm dynamics of the solutions to (1.1). More interestingly, the limit procedure is automatically built-in, for we do not let any parameter go to zero artificially (e.g. like in [7]). As far as we can say, this is the first considered instance of limiting procedure not induced by an actual limit.

The first part of this work is devoted to the general theory of attractors for processes in time-dependent spaces, specifically developed in $[1,2,8]$ to handle evolution problems where the coefficients of the differential operators depend explicitly on time. Due to the presence of time-dependent terms at a functional level, the standard theory of attractors fails to capture the dissipation mechanism involved in the dynamical system, and thus it is not suitable to study its longterm behavior. The phenomenon is quite evident in the model equation (1.1) under consideration, where what is really dissipated is the weak energy

$$
E(t)=\int_{\Omega}|\nabla u(\boldsymbol{x}, t)|^{2} \mathrm{~d} \boldsymbol{x}+\varepsilon(t) \int_{\Omega}\left|u_{t}(\boldsymbol{x}, t)\right|^{2} \mathrm{~d} \boldsymbol{x} .
$$

Hence, it seems convenient to frame the equation in the usual Sobolev space

$$
z_{t}=H_{0}^{1}(\Omega) \times L^{2}(\Omega),
$$

but endowed with the norm

$$
\|(u, v)\|_{\mathcal{L}_{t}}^{2}=\|u\|_{H_{0}^{1}}^{2}+\varepsilon(t)\|v\|_{L^{2}}^{2},
$$

depending on time through the weight function $\varepsilon(t)$. This leads us quite naturally to adopt a new point of view, namely, to describe the solution operator as a family of maps acting on the time-dependent family of spaces $\left\{\mathcal{Z}_{t}\right\}$. After a brief review of the main definitions of processes on time-dependent spaces and their attractors, we supplement the general theory with two new results. The first gives the structure of the time-dependent attractor in terms of complete bounded trajectories of the system (see Theorem 3.2). The second result (Theorem 4.2) addresses in an abstract form the main question stated in the introduction; precisely, when the original model formally collapses into an autonomous system as $t \rightarrow \infty$, we provide sufficient conditions in order for $A_{t}$ to be close (in a suitable sense) to the global attractor of the limiting equation.

As a concrete application of the abstract result, in the second part of the paper we focus on the wave equation (1.1) with time-dependent speed of propagation and its relationships with the parabolic heat model (1.2). In particular, the closeness between the time-dependent global attractor $A_{t}$ associated with (1.1) and the global attractor $A_{\infty}$ associated with (1.2) is shown to occur in terms of Hausdorff semidistance.

\section{Time-dependent global attractors: a review}

We begin by recalling some basic definitions and results from $[1,2,8]$ about processes on time-dependent spaces. For $t \in \mathbb{R}$, let $\mathcal{Z}_{t}$ be a family of normed spaces. We consider a two-parameter family of operators

$$
U(t, \tau): Z_{\tau} \rightarrow Z_{t},
$$

depending on $t \geq \tau \in \mathbb{R}$, satisfying the following properties:

(i) $U(\tau, \tau)$ is the identity map on $\mathfrak{Z}_{\tau}$ for every $\tau$;

(ii) $U(t, \tau) U(\tau, s)=U(t, s)$ for every $t \geq \tau \geq s$.

The family $U(t, \tau)$ is called a process.

We now give the definition of global attractor in this context. First, we introduce a suitable notion of uniform boundedness.

Definition 2.1. A family $\mathfrak{C}=\left\{C_{t}\right\}_{t \in \mathbb{R}}$ of bounded sets $C_{t} \subset \mathcal{Z}_{t}$ is called uniformly bounded if there exists $R>0$ such that

$$
C_{t} \subset\left\{\zeta \in \mathcal{Z}_{t}:\|\zeta\|_{\mathcal{Z}_{t}} \leq R\right\}, \quad \forall t \in \mathbb{R} .
$$

Analogously to [9], the global attractor in this framework can be defined as in [1], through the property of being the minimal set among the compact attracting ones.

Definition 2.2. The time-dependent global attractor for $U(t, \tau)$ is the smallest family $\mathfrak{A}=\left\{A_{t}\right\}_{t \in \mathbb{R}}$ such that

(i) each $A_{t}$ is compact in $Z_{t}$;

(ii) $\mathfrak{A}$ is pullback attracting, i.e. it is uniformly bounded and the limit ${ }^{1}$

$$
\lim _{\tau \rightarrow-\infty} \delta_{\mathcal{Z}_{t}}\left(U(t, \tau) C_{\tau}, A_{t}\right)=0
$$

holds for every uniformly bounded family $\mathfrak{C}=\left\{C_{t}\right\}_{t \in \mathbb{R}}$ and every fixed $t \in \mathbb{R}$.

1 For any normed space $\mathcal{Z}$, the Hausdorff semidistance of two (nonempty) sets $B, C \subset \mathcal{Z}$ is given by

$$
\boldsymbol{\delta}_{\mathcal{Z}}(B, C)=\sup _{x \in B} \operatorname{dist}_{\mathcal{Z}}(x, C)=\sup _{x \in B} \inf _{y \in C}\|x-y\|_{\mathcal{Z}} .
$$


Remark 2.3. By virtue of [1, Theorem 4.2] $\mathfrak{A}$ exists and is unique if and only if the set

$\left\{\mathfrak{K}=\left\{K_{t}\right\}_{t \in \mathbb{R}}: K_{t} \subset \mathcal{Z}_{t}\right.$ compact, $\mathfrak{K}$ pullback attracting $\}$

is not empty.

The next issue is the invariance of the attractor.

Definition 2.4. A family $\mathfrak{C}=\left\{C_{t}\right\}_{t \in \mathbb{R}}$ is called invariant if

$U(t, \tau) C_{\tau}=C_{t}, \quad \forall t \geq \tau, \tau \in \mathbb{R}$.

Although such a property is not required in Definition 2.2, the time-dependent global attractor turns out to be invariant as soon as the process satisfies some weak continuity-like properties (see [1, Theorem 5.6]). In particular, the following is true.

Theorem 2.5. If $U(t, \tau)$ is a strongly continuous process which possesses the time-dependent global attractor $\mathfrak{A}$, then $\mathfrak{A}$ is invariant.

Recall that a process is called strongly continuous if $U(t, \tau)$ is a continuous map from $Z_{\tau}$ to $Z_{t}$ for any pair of fixed times $t \geq \tau$.

Noticeably, whenever invariant, the time-dependent global attractor is maximal in the class of bounded invariant families, as a consequence of the next proposition.

Proposition 2.6. If $\mathfrak{C}=\left\{C_{t}\right\}_{t \in \mathbb{R}}$ is any uniformly bounded invariant family, then

$$
\mathfrak{C} \subset \mathfrak{A} \text {. }
$$

Proof. Since $\mathfrak{A}$ is pullback attracting and $\mathfrak{C}$ is uniformly bounded, then

$$
\lim _{\tau \rightarrow-\infty} \delta_{Z_{t}}\left(U(t, \tau) C_{\tau}, A_{t}\right)=0 .
$$

But $U(t, \tau) C_{\tau}=C_{t}$, hence

$$
\delta_{Z_{t}}\left(C_{t}, A_{t}\right)=0 \text {, }
$$

which implies $C_{t} \subset \bar{C}_{t} \subset \bar{A}_{t}=A_{t}$ for all $t$ (the bar standing for closure in $\mathcal{Z}_{t}$ ).

\section{Complete bounded trajectories}

Similarly to the classical case, the time-dependent global attractor can be characterized as sections of the set of complete bounded trajectories.

Definition 3.1. A function $z: t \mapsto z(t) \in \mathcal{Z}_{t}$ is a complete bounded trajectory (свт) of $U(t, \tau)$ if and only if

$$
\sup _{t \in \mathbb{R}}\|z(t)\|_{z_{t}}<\infty
$$

and

$$
z(t)=U(t, \tau) z(\tau), \quad \forall t \geq \tau, \tau \in \mathbb{R}
$$

Theorem 3.2. Let $\mathfrak{A}=\left\{A_{t}\right\}_{t \in \mathbb{R}}$ be the time-dependent global attractor of $U(t, \tau)$. If $\mathfrak{A}$ is invariant, then

$$
A_{t}=\left\{z(t) \in \mathcal{Z}_{t}: z \text { свт of } U(t, \tau)\right\} .
$$

Accordingly, we can write

$$
\mathfrak{A}=\left\{z: t \mapsto z(t) \in \mathcal{Z}_{t} \text { with } z \text { свт of } U(t, \tau)\right\} .
$$

Proof. Let us denote

$$
\tilde{\mathfrak{A}}=\left\{\tilde{A}_{t}\right\}_{t \in \mathbb{R}} \quad \text { with } \tilde{A}_{t}=\left\{z(t) \in \mathcal{Z}_{t}: z \text { CBT of } U(t, \tau)\right\} .
$$

We have to prove the set equality

$$
\mathfrak{A}=\tilde{\mathfrak{A}} \text {. }
$$

Let us first show that $\tilde{\mathfrak{A}} \subset \mathfrak{A}$. To this aim, let $z(\cdot)$ be any fixed свт of $U(t, \tau)$. In particular, the family

$$
\mathfrak{B}_{z}=\{z(t)\}_{t \in \mathbb{R}}
$$

is uniformly bounded and invariant. Hence, by Proposition 2.6,

$\mathfrak{B}_{z} \subset \mathfrak{A} \Rightarrow \tilde{\mathfrak{A}} \subset \mathfrak{A}$. 
To see the converse, let $\zeta \in A_{s}$ for a fixed $s$. Using the invariance of $\mathfrak{A}$, we build in a recursive way a sequence $\zeta_{n}$ (with $\zeta_{0}=\zeta$ ) such that

$$
\zeta_{n}=U(s-n, s-n-1) \zeta_{n+1} .
$$

Notice that $\zeta_{n} \in A_{s-n}$ for all $n$. Define then

$$
z(t)=U(t, s-n) \zeta_{n}, \quad \text { for } t \geq s-n .
$$

The definition is well posed, since if $t \geq s-m$ and we assume, for instance, $n \geq m$, then

$$
U(t, s-n) \zeta_{n}=U(t, s-m) U(s-m, s-n) \zeta_{n}=U(t, s-m) \zeta_{m} .
$$

Since $z(t) \in A_{t}$ and $\mathfrak{A}$ is uniformly bounded, then

$$
\sup _{t \in \mathbb{R}}\|z(t)\|_{\mathcal{Z}_{t}}<\infty \text {. }
$$

Finally, if $t \geq \tau$, choosing $n$ such that $\tau \geq s-n$ we have

$$
z(t)=U(t, s-n) \zeta_{n}=U(t, \tau) U(\tau, s-n) \zeta_{n}=U(t, \tau) z(\tau),
$$

proving that $z$ is a СвT of $U(t, \tau)$, and, in particular,

$$
z(s) \in \tilde{A}_{s} .
$$

Exploiting the equality $\zeta=z(s)$, we conclude that $A_{s} \subset \tilde{A}_{s}$, which yields the sought family inclusion $\mathfrak{A} \subset \tilde{\mathfrak{A}}$.

\section{Upper semicontinuity of the attractor}

In what follows, we will focus on the particular, albeit significant, case of a process $U(t, \tau)$ acting on a family of spaces $\left\{\mathcal{Z}_{t}\right\}_{t \in \mathbb{R}}$ of the form

$$
z_{t}=x \times y_{t}
$$

where $\mathcal{X}$ is a normed space and $\left\{y_{t}\right\}_{t \in \mathbb{R}}$ is a family of normed spaces, endowed with the product norm

$$
\|(\xi, \eta)\|_{\mathcal{Z}_{t}}^{2}=\|\xi\|_{x}^{2}+\|\eta\|_{y_{t}}^{2} \text {. }
$$

\subsection{Notation}

We denote by $\Pi_{t}: Z_{t} \rightarrow \mathcal{X}$ the projection on the first component of $\mathcal{Z}_{t}$, namely,

$$
\Pi_{t}(\xi, \eta)=\xi \text {. }
$$

Accordingly, if $C_{t} \subset Z_{t}$, then

$$
\Pi_{t} C_{t}=\left\{\xi \in \mathcal{X}:(\xi, \eta) \in C_{t}\right\} .
$$

In a similar manner, if $\mathfrak{C}=\left\{C_{t}\right\}_{t \in \mathbb{R}}$, we set

$$
\Pi \mathfrak{C}=\left\{\Pi_{t} C_{t}\right\}_{t \in \mathbb{R}} .
$$

\subsection{General assumptions}

We suppose that our process $U(t, \tau)$ possesses the time-dependent global attractor $\mathfrak{A}=\left\{A_{t}\right\}_{t \in \mathbb{R}}$, which is assumed to be invariant as well. Observe that, by Theorem 3.2, we can write

$$
\Pi \mathfrak{A}=\{x: \mathbb{R} \rightarrow \mathcal{X} \text { such that } x=\Pi z \text { with } z \text { свт of } U(t, \tau)\} .
$$

In addition, let

$$
S(t): X \rightarrow X, \quad t \geq 0
$$

be a semigroup acting on $\mathcal{X}$, possessing the (classical) global attractor $A_{\infty} \subset \mathcal{X}$.

Recall that the global attractor of the semigroup $S(t)$ is by definition the unique compact set $A_{\infty} \subset \mathcal{X}$ which is simultaneously invariant, i.e.

$$
S(t) A_{\infty}=A_{\infty}, \quad \forall t \geq 0,
$$

and attracting the bounded subsets of $\mathcal{X}$ with respect to the Hausdorff semidistance in $\mathcal{X}$ (see e.g. [3-6]).

Remark 4.1. It is well known that, for any fixed $s \in \mathbb{R}$, the global attractor of $S(t)$ has the form

$$
A_{\infty}=\{w(s): w \text { свт of } S(t)\},
$$


where $w: \mathbb{R} \rightarrow \mathcal{X}$ is called a свт of $S(t)$ if

$$
\sup _{t \in \mathbb{R}}\|w(t)\|_{x}<\infty \text { and } w(t+\tau)=S(t) w(\tau),
$$

for all $t \geq 0$ and $\tau \in \mathbb{R}$. Besides, $w \in \mathcal{C}(\mathbb{R}, \mathcal{X})$ whenever the map $t \mapsto S(t) \xi$ belongs to $\mathcal{C}\left(\mathbb{R}^{+}, \mathcal{X}\right)$ for all $\xi \in \mathcal{X}$.

\subsection{The abstract result}

We are now ready to state the theorem on the convergence of $A_{t}$ to $A_{\infty}$.

Theorem 4.2. Assume that, for any sequence $z_{n}=\left(x_{n}, y_{n}\right)$ of свт of the process $U(t, \tau)$ and any $t_{n} \rightarrow \infty$, there exist a свт $w$ of $S(t)$ and $s \in \mathbb{R}$ for which

$$
\left\|x_{n}\left(s+t_{n}\right)-w(s)\right\|_{x} \rightarrow 0
$$

as $n \rightarrow \infty$, up to a subsequence. Then

$$
\lim _{t \rightarrow \infty} \delta_{x}\left(\Pi_{t} A_{t}, A_{\infty}\right)=0 .
$$

Proof. By contradiction, let there exist $v>0$ and two sequences $t_{n} \rightarrow \infty$ and $\zeta_{n} \in \Pi_{t_{n}} A_{t_{n}}$ such that

$$
\inf _{\xi \in A_{\infty}}\left\|\zeta_{n}-\xi\right\|_{x} \geq v
$$

In light of Theorem 3.2, for all $n$ there is a свт $z_{n}=\left(x_{n}, y_{n}\right)$ of $U(t, \tau)$ satisfying

$$
\zeta_{n}=x_{n}\left(t_{n}\right) \text {. }
$$

If we define the function $\hat{z}_{n}=\left(\hat{x}_{n}, \hat{y}_{n}\right)$ by the rule

$$
\hat{z}_{n}(t)=z_{n}(t-s)
$$

then $\hat{z}_{n}$ is still a свт of $U(t, \tau)$ (note that $\hat{z}_{n}(t) \in A_{t-s}$ ). Besides,

$$
\zeta_{n}=\hat{x}_{n}\left(s+t_{n}\right) \text {. }
$$

Therefore, by assumption, we find a Свт $w$ of $S(t)$ such that

$$
\left\|\zeta_{n}-w(s)\right\|_{X}=\left\|\hat{x}_{n}\left(s+t_{n}\right)-w(s)\right\|_{X} \rightarrow 0
$$

up to a subsequence. Since $w(s) \in A_{\infty}$ by Remark 4.1, this violates (4.1).

\section{Wave equations with time-dependent speed of propagation}

Let $\Omega \subset \mathbb{R}^{3}$ be a bounded domain with smooth boundary $\partial \Omega$. For any initial time $\tau \in \mathbb{R}$, we consider as in [1] the evolution equation in the unknown $u=u(\boldsymbol{x}, t): \Omega \times[\tau, \infty) \rightarrow \mathbb{R}$

$$
\varepsilon u_{t t}+\alpha u_{t}-\Delta u+f(u)=g
$$

subject to the homogeneous Dirichlet boundary condition

$$
u(\boldsymbol{x}, t)_{\mid \boldsymbol{x} \in \partial \Omega}=0
$$

and the initial conditions

$$
u(\boldsymbol{x}, \tau)=a(\boldsymbol{x}) \text { and } u_{t}(\boldsymbol{x}, \tau)=b(\boldsymbol{x}),
$$

where $a, b: \Omega \rightarrow \mathbb{R}$ are assigned data. Here, the damping coefficient $\alpha$ is a strictly positive constant while $\varepsilon=\varepsilon(t)$ is a function of $t$.

\subsection{General assumptions}

Let $\varepsilon \in \mathcal{C}^{1}(\mathbb{R})$ be a positive decreasing bounded function with bounded derivative satisfying

$$
\lim _{t \rightarrow \infty} \varepsilon(t)=0
$$

Besides, let $f \in \mathcal{C}^{2}(\mathbb{R})$ with $f(0)=0$ satisfy the growth bound

$$
\left|f^{\prime \prime}(u)\right| \leq c(1+|u|),
$$

for some $c \geq 0$, along with the dissipation condition

$$
\liminf _{|s| \rightarrow \infty} \frac{f(s)}{s}>-\lambda_{1}
$$


where $\lambda_{1}>0$ is the first eigenvalue of the strictly positive Dirichlet operator

$-\Delta$ with domain $\mathfrak{D}(-\Delta)=H^{2}(\Omega) \cap H_{0}^{1}(\Omega) \Subset L^{2}(\Omega)$.

Finally, the time-independent external source $g=g(\boldsymbol{x})$ is taken in $L^{2}(\Omega)$.

\subsection{Functional setting and notation}

We set $\mathrm{H}=L^{2}(\Omega)$, with inner product $\langle\cdot, \cdot\rangle$ and norm $\|\cdot\|$. For $\sigma \in \mathbb{R}$, we define the hierarchy of (compactly) nested Hilbert spaces

$$
\mathrm{H}^{\sigma}=\mathfrak{D}\left((-\Delta)^{\frac{\sigma}{2}}\right), \quad\langle u, v\rangle_{\sigma}=\left\langle(-\Delta)^{\frac{\sigma}{2}} u,(-\Delta)^{\frac{\sigma}{2}} v\right\rangle, \quad\|u\|_{\sigma}=\left\|(-\Delta)^{\frac{\sigma}{2}} u\right\| .
$$

The symbol $\sigma$ is always omitted whenever zero. Then, for all $t \in \mathbb{R}$, we introduce the time-dependent spaces

$$
z_{t}=\mathrm{H}^{1} \times \mathrm{H} \text {, }
$$

endowed with the time-dependent product norms

$$
\|(u, v)\|_{\mathcal{Z}_{t}}^{2}=\|u\|_{1}^{2}+\varepsilon(t)\|v\|^{2} .
$$

Note that $\mathcal{Z}_{t}$ are all the same as linear spaces. Besides, since $\varepsilon(\cdot)$ is a decreasing function of $t$, for every $(u, v) \in \mathrm{H}^{1} \times \mathrm{H}$ and $t \geq \tau \in \mathbb{R}$ we have

$$
\|(u, v)\|_{\mathcal{Z}_{t}}^{2} \leq\|(u, v)\|_{\mathcal{Z}_{\tau}}^{2} \leq \max \left\{1, \frac{\varepsilon(\tau)}{\varepsilon(t)}\right\}\|(u, v)\|_{\mathcal{Z}_{t}}^{2} .
$$

Thus, the norms in $\mathcal{Z}_{t}$ and $\mathcal{Z}_{\tau}$ are equivalent for any fixed $t, \tau \in \mathbb{R}$, but the equivalence constant blows up when $t \rightarrow \infty$.

\subsection{The process and its attractor}

According to [1], problem (5.1)-(5.3) generates a process

$$
U(t, \tau): \mathfrak{Z}_{\tau} \rightarrow Z_{t}
$$

by the rule

$$
U(t, \tau) \zeta=\left(u(t), u_{t}(t)\right),
$$

where $\left(u(t), u_{t}(t)\right)$ is the unique solution to (5.1)-(5.2) with initial datum $\zeta=(a, b)$ taken at the initial time $t=\tau$. Besides, for every pair of initial data $\zeta_{l}=\left(a_{l}, b_{l}\right) \in \mathcal{Z}_{\tau}$ such that $\left\|\zeta_{l}\right\|_{\mathcal{Z}_{\tau}} \leq R$, the difference of the corresponding solutions satisfies

$$
\left\|U(t, \tau) \zeta_{1}-U(t, \tau) \zeta_{2}\right\|_{\mathcal{Z}_{t}} \leq \mathrm{e}^{K(t-\tau)}\left\|\zeta_{1}-\zeta_{2}\right\|_{\mathcal{Z}_{\tau}}, \quad \forall t \geq \tau
$$

for some constant $K=K(R) \geq 0$. Therefore, $U(t, \tau)$ is strongly continuous.

The main result concerning the asymptotic behavior of the process $U(t, \tau)$ is contained in [1, Theorem 11.1].

Theorem 5.1. The process $U(t, \tau)$ possesses an invariant time-dependent global attractor $\mathfrak{A}=\left\{A_{t}\right\}_{t \in \mathbb{R}}$. Besides, for every $\tau \in \mathbb{R}$, the uniform estimate

$$
\sup _{z \in A_{\tau}} \sup _{t \geq \tau}\left[\|u(t)\|_{2}+\varepsilon(t)\left\|u_{t}(t)\right\|_{1}^{2}+\int_{\tau}^{\infty}\left\|u_{t}(y)\right\|^{2} \mathrm{~d} y\right] \leq c
$$

holds for some constant $c=c(\mathfrak{A}) \geq 0$ independent of $\tau$.

Remark 5.2. Since it is invariant, by Theorem 3.2 we conclude that the time-dependent global attractor coincides with the set of all complete bounded trajectories of $U(t, \tau)$, i.e.

$$
\mathfrak{A}=\left\{z: t \mapsto z(t)=\left(u(t), u_{t}(t)\right) \in \mathcal{Z}_{t} \text { with } z \text { свт of } U(t, \tau)\right\} .
$$

\section{Asymptotic structure of the attractor}

In the same spirit of the abstract Theorem 4.2, we now wish to investigate the relationship between the time-dependent global attractor of $U(t, \tau)$ and the global attractor of the limit equation

$$
\alpha u_{t}-\Delta u+f(u)=g \text {, }
$$

formally corresponding to (5.1) when $t \rightarrow \infty$.

Within our assumptions on $f$ and $g$, it is well known that Eq. (6.1) with the homogeneous Dirichlet boundary condition generates a strongly continuous semigroup (see e.g. $[3,6]$ )

$$
S(t): \mathrm{H}^{1} \rightarrow \mathrm{H}^{1}
$$


by the rule

$$
S(t) a=u(t),
$$

where $u(t)$ is the unique solution to (6.1) with initial condition

$$
u(\boldsymbol{x}, 0)=a(\boldsymbol{x}) .
$$

Furthermore, $S(t)$ admits the (classical) global attractor $A_{\infty}$ which turns out to be a bounded subset of $\mathrm{H}^{2}$. From Remark 4.1, we also know that, for any fixed $s \in \mathbb{R}$,

$$
A_{\infty}=\{w(s): w \text { свт of } S(t)\} .
$$

The main result of this paper establishes the asymptotic closeness of the time-dependent global attractor $\mathfrak{A}=\left\{A_{t}\right\}_{t \in \mathbb{R}}$ of the process $U(t, \tau)$ generated by (5.1) and the global attractor $A_{\infty}$ of the semigroup $S(t)$ generated by (6.1).

Theorem 6.1. The following limit holds:

$$
\lim _{t \rightarrow \infty} \delta_{\mathrm{H}^{1}}\left(\Pi_{t} A_{t}, A_{\infty}\right)=0 .
$$

The proof is an application of the abstract Theorem 4.2, whose hypotheses are verified (even in greater generality) in the next lemma. In what follows, $c=c(\mathfrak{A})$ will always stand for a generic positive constant depending only on $\mathfrak{A}=\left\{A_{t}\right\}_{t \in \mathbb{R}}$.

Lemma 6.2. For any sequence $z_{n}=\left(u_{n}, \partial_{t} u_{n}\right)$ of свт of the process $U(t, \tau)$ and any $t_{n} \rightarrow \infty$, there exists a CBT $w$ of $S(t)$ such that, for every $T>0$,

$$
\sup _{t \in[-T, T]}\left\|u_{n}\left(t+t_{n}\right)-w(t)\right\|_{\mathrm{H}^{1}} \rightarrow 0
$$

as $n \rightarrow \infty$, up to a subsequence.

Proof. Owing to (5.5), for every $T>0$ we deduce the boundedness of the sequence $u_{n}\left(\cdot+t_{n}\right)$ in the space

$$
L^{\infty}\left(-T, T ; \mathrm{H}^{2}\right) \cap W^{1,2}(-T, T ; \mathrm{H}),
$$

and a direct application of corollary 4 in [10] shows that $u_{n}\left(\cdot+t_{n}\right)$ is relatively compact in $\mathcal{C}\left([-T, T], \mathrm{H}^{1}\right)$, for every $T>0$. Hence there exists a function

$$
w: \mathbb{R} \rightarrow \mathrm{H}^{1}
$$

such that, up to a subsequence, the convergence

$$
u_{n}\left(\cdot+t_{n}\right) \rightarrow w(\cdot)
$$

holds in the sense of (6.2). In particular, $w \in \mathcal{C}\left(\mathbb{R}, \mathrm{H}^{1}\right)$. Besides, recalling (5.5),

$$
\sup _{t \in \mathbb{R}}\|w(t)\|_{1} \leq c .
$$

We are left to show that $w$ solves (6.1). To this end, we define

$$
v_{n}(t)=u_{n}\left(t+t_{n}\right) \text { and } \varepsilon_{n}(t)=\varepsilon\left(t+t_{n}\right),
$$

and we rewrite Eq. (5.1) for $u_{n}$ in the form

$$
\alpha \partial_{t} v_{n}=-\varepsilon_{n} \partial_{t t} v_{n}+\Delta v_{n}-f\left(v_{n}\right)+g .
$$

We first prove that the sequence $\varepsilon_{n} \partial_{t t} v_{n}$ converges to zero in the distributional sense. Indeed, for every fixed $T>0$ and every smooth $\mathrm{H}$-valued function $\varphi$ supported on $(-T, T)$, we have

$$
\int_{-T}^{T} \varepsilon_{n}(t)\left\langle\partial_{t t} v_{n}(t), \varphi(t)\right\rangle \mathrm{d} t=-\int_{-T}^{T} \varepsilon_{n}(t)\left\langle\partial_{t} v_{n}(t), \partial_{t} \varphi(t)\right\rangle \mathrm{d} t-\int_{-T}^{T} \varepsilon_{n}^{\prime}(t)\left\langle\partial_{t} v_{n}(t), \varphi(t)\right\rangle \mathrm{d} t .
$$

Consequently, exploiting again (5.5),

$$
\begin{aligned}
\left|\int_{-T}^{T} \varepsilon_{n}(t)\left\langle\partial_{t t} v_{n}(t), \varphi(t)\right\rangle \mathrm{d} t\right| & \leq c \int_{-T}^{T} \sqrt{\varepsilon_{n}(t)} \sqrt{\varepsilon_{n}(t)}\left\|\partial_{t} v_{n}(t)\right\|_{1} \mathrm{~d} t+c \int_{-T}^{T} \frac{\left|\varepsilon_{n}^{\prime}(t)\right|}{\sqrt{\varepsilon_{n}(t)}} \sqrt{\varepsilon_{n}(t)}\left\|\partial_{t} v_{n}(t)\right\|_{1} \mathrm{~d} t \\
& \leq c \int_{-T}^{T} \sqrt{\varepsilon_{n}(t)} \mathrm{d} t+c \int_{-T}^{T} \frac{\left|\varepsilon_{n}^{\prime}(t)\right|}{\sqrt{\varepsilon_{n}(t)}} \mathrm{d} t \\
& \leq c T \sup _{t \in[-T, T]} \sqrt{\varepsilon_{n}(t)}+c\left(\sqrt{\varepsilon_{n}(T)}-\sqrt{\varepsilon_{n}(-T)}\right),
\end{aligned}
$$


where the generic constant $c>0$ depends also on $\varphi$. Since

$$
\lim _{n \rightarrow \infty}\left[\sup _{t \in[-T, T]} \varepsilon_{n}(t)\right]=0,
$$

we reach the desired conclusion

$$
\lim _{n \rightarrow \infty} \int_{-T}^{T} \varepsilon_{n}(t)\left\langle\partial_{t t} v_{n}(t), \varphi(t)\right\rangle \mathrm{d} t=0 .
$$

Now, taking into account (5.4), the convergence (6.2) yields (up to a subsequence)

$$
\Delta v_{n}-f\left(v_{n}\right) \rightarrow \Delta w-f(w)
$$

in the topology of $L^{\infty}\left(-T, T ; \mathrm{H}^{-1}\right)$, for every $T>0$. At the same time, the convergence

$$
\partial_{t} v_{n} \rightarrow w_{t}
$$

holds (again up to a subsequence) in the distributional sense. Therefore, we end up with the equality

$$
\alpha w_{t}-\Delta w+f(w)=g,
$$

which, together with (6.3), proves that $w$ is a свт of the semigroup $S(t)$.

Proof of Theorem 6.1. Thanks to Lemma 6.2, we can readily apply Theorem 4.2 with

$$
x=\mathrm{H}^{1} \text { and } y_{t}=\mathrm{H},
$$

the latter space endowed with the norm

$$
\|\cdot\| y_{t}=\sqrt{\varepsilon(t)}\|\cdot\| .
$$

This finishes the proof of Theorem 6.1.

\section{Further regularity of the attractor}

The aim of this final section is to establish further regularity properties for the trajectories lying on the attractor, within the additional assumption

$$
\beta:=\inf _{t \in \mathbb{R}}\left[2 \alpha+\varepsilon^{\prime}(t)\right]>0 .
$$

Namely, denoting

$$
\Pi \mathfrak{A}=\left\{u: \mathbb{R} \rightarrow \mathrm{H}^{1} \text { such that } u=\Pi z \text { with } z \text { свт of } U(t, \tau)\right\},
$$

we prove that, for every $u \in \Pi \mathfrak{A}$, the uniform estimate for $\left\|u_{t}\right\|_{1}$ provided by (5.5) is in fact independent of $\varepsilon$. Again, we will denote by $c=c(\mathfrak{A})$ a generic positive constant.

Theorem 7.1. Let (7.1) hold. Then we have the uniform estimate

$$
\sup _{u \in \Pi \mathfrak{A}} \sup _{t \in \mathbb{R}}\left[\left\|u_{t}(t)\right\|_{1}+\sqrt{\varepsilon(t)}\left\|u_{t t}(t)\right\|\right] \leq c .
$$

The proof of Theorem 7.1 is based on the following lemma.

Lemma 7.2. The uniform bound

$$
\sup _{u \in \Pi \mathfrak{A}}\left[\left\|u_{t}(t)\right\|_{1}^{2}+\varepsilon(t)\left\|u_{t t}(t)\right\|^{2}\right] \leq \frac{c}{\varepsilon^{2}(\tau)} \mathrm{e}^{-v(t-\tau)}+c
$$

holds for some $v>0$ and every $t \geq \tau$.

Proof. Let $u \in \Pi \mathfrak{A}$ and let $\tau \in \mathbb{R}$ be fixed. Then $u$ solves (5.1)-(5.2) with initial conditions $\left(u(\tau), u_{t}(\tau)\right)$ for every $t \geq \tau$. Differentiating (5.1) in time, the function $q=u_{t}$ satisfies the equation

$$
\varepsilon q_{t t}+\left(\alpha+\varepsilon^{\prime}\right) q_{t}-\Delta q=N(u)
$$

with the homogeneous Dirichlet boundary condition, having set

$$
N(u)=-f^{\prime}(u) u_{t} .
$$

Accordingly, the initial data read

$$
q(\tau)=u_{t}(\tau) \quad \text { and } \quad q_{t}(\tau)=\frac{1}{\varepsilon(\tau)}\left[g-f(u(\tau))-\alpha u_{t}(\tau)+\Delta u(\tau)\right] .
$$


Since $\left(u(\tau), u_{t}(\tau)\right) \in A_{\tau}$, making use of (5.5) we deduce the bound

$$
\left\|\left(q(\tau), q_{t}(\tau)\right)\right\|_{\mathcal{L}_{\tau}} \leq \frac{c}{\varepsilon(\tau)}
$$

Besides, from the integral estimate in (5.5) and the Agmon inequality, we see at once that

$$
\int_{\tau}^{\infty}\|N(u(y))\|^{2} \mathrm{~d} y \leq c .
$$

Multiplying the equation times $2 q_{t}$, we get

$$
\frac{\mathrm{d}}{\mathrm{d} t}\left[\|q\|_{1}^{2}+\varepsilon\left\|q_{t}\right\|^{2}\right]+\left(2 \alpha+\varepsilon^{\prime}\right)\left\|q_{t}\right\|^{2}=2\left\langle N(u), q_{t}\right\rangle,
$$

while a further multiplication by $2 \delta q$, for $\delta>0$ to be fixed later, yields

$$
\frac{\mathrm{d}}{\mathrm{d} t}\left[\delta \alpha\|q\|^{2}+2 \delta \varepsilon\left\langle q, q_{t}\right\rangle\right]+2 \delta\|q\|_{1}^{2}-2 \delta \varepsilon\left\|q_{t}\right\|^{2}=2 \delta\langle N(u), q\rangle .
$$

Collecting the two differential identities, we end up with

$$
\frac{\mathrm{d}}{\mathrm{d} t} \Lambda+2 \delta\|q\|_{1}^{2}+\left(2 \alpha+\varepsilon^{\prime}-2 \delta \varepsilon\right)\left\|q_{t}\right\|^{2}=2\left\langle N(u), q_{t}+\delta q\right\rangle,
$$

where the functional

$$
\Lambda(t)=\|q(t)\|_{1}^{2}+\delta \alpha\|q(t)\|^{2}+\varepsilon(t)\left\|q_{t}(t)\right\|^{2}+2 \delta \varepsilon(t)\left\langle q(t), q_{t}(t)\right\rangle
$$

satisfies, for $\delta>0$ small,

$$
\frac{1}{C}\left\|\left(q(t), q_{t}(t)\right)\right\|_{\mathcal{Z}_{t}}^{2} \leq \Lambda(t) \leq C\left\|\left(q(t), q_{t}(t)\right)\right\|_{\mathcal{Z}_{t}}^{2},
$$

for some $C>1$. Indeed, as

$$
L:=\sup _{t \in \mathbb{R}} \varepsilon(t)<\infty,
$$

we can write

$$
2 \delta \varepsilon\left|\left\langle q_{t}, q\right\rangle\right| \leq \frac{\varepsilon}{2}\left\|q_{t}\right\|^{2}+2 L \delta^{2}\|q\|^{2} \leq \frac{\varepsilon}{2}\left\|q_{t}\right\|^{2}+\delta \alpha\|q\|^{2},
$$

provided that $\delta>0$ is small enough. Moreover, in light of assumption (7.1), and possibly choosing a smaller $\delta>0$, we have

$$
\inf _{t \in \mathbb{R}}\left[2 \alpha+\varepsilon^{\prime}(t)-2 \delta \varepsilon(t)\right] \geq \beta-2 \delta L \geq \frac{\beta}{2}>0 .
$$

Finally, controlling via standard computations the term $2\left\langle N(u), q_{t}+\delta q\right\rangle$, we find $v>0$, depending only on $\delta$ and $\beta$, such that

$$
\frac{\mathrm{d}}{\mathrm{d} t} \Lambda+v \Lambda \leq \kappa\|N(u)\|^{2}
$$

for some $\kappa=\kappa(v)>0$. At this point, keeping in mind (7.4), the Gronwall Lemma entails

$$
\Lambda(t) \leq \Lambda(\tau) \mathrm{e}^{-v(t-\tau)}+c .
$$

Thus, exploiting (7.5) and estimating $\Lambda(\tau)$ through (7.3), we are led to

$$
\left\|u_{t}(t)\right\|_{1}^{2}+\varepsilon(t)\left\|u_{t t}(t)\right\|^{2} \leq \frac{c}{\varepsilon^{2}(\tau)} \mathrm{e}^{-v(t-\tau)}+c
$$

for every $t \geq \tau$, as claimed.

Proof of Theorem 7.1. Let $z(t)=\left(u(t), u_{t}(t)\right)$ be any given свт of the process $U(t, \tau)$. By the invariance of the attractor, for any $\tau \leq t$ there exists $\zeta \in A_{\tau}$ such that

$$
z(t)=U(t, \tau) \zeta .
$$

Hence, by Lemma 7.2,

$$
\left\|u_{t}(t)\right\|_{1}^{2}+\varepsilon(t)\left\|u_{t t}(t)\right\|^{2} \leq \frac{c}{\varepsilon^{2}(\tau)} \mathrm{e}^{-v(t-\tau)}+c .
$$

Since $\varepsilon$ is bounded at $-\infty$, the required estimate follows by taking the limit $\tau \rightarrow-\infty$. 


\section{Acknowledgment}

The authors are grateful to the anonymous referee for useful remarks and comments.

\section{References}

[1] M. Conti, V. Pata, R. Temam, Attractors for processes on time-dependent spaces. Applications to wave equations, J. Differential Equations 255 (2013) $1254-1277$.

[2] F. Di Plinio, G.S. Duane, R. Temam, Time dependent attractor for the oscillon equation, Discrete Contin. Dyn. Syst. 29 (2011) $141-167$.

[3] A.V. Babin, M.I. Vishik, Attractors of Evolution Equations, North-Holland, Amsterdam, 1992.

[4] J.K. Hale, Asymptotic Behavior of Dissipative Systems, American Mathematical Society, Providence, 1988.

[5] A. Haraux, Systèmes Dynamiques Dissipatifs et Applications, Masson, Paris, 1991.

[6] R. Temam, Infinite-Dimensional Dynamical Systems in Mechanics and Physics, Springer, New York, 1997.

[7] J.K. Hale, G. Raugel, Upper semicontinuity of the attractor for a singularly perturbed hyperbolic equation, J. Differential Equations 73 (1988) $197-214$.

[8] F. Di Plinio, G.S. Duane, R. Temam, The 3-dimensional oscillon equation, Boll. Unione Mat. Ital. (9) 5 (2012) 19-53.

[9] V.V Chepyzhov, M. Conti, V. Pata, A minimal approach to the theory of global attractors, Discrete Contin. Dyn. Syst. 32 (2012) 2079-2088.

[10] J. Simon, Compact sets in the space $L^{p}(0, T ; B)$, Ann. Mat. Pura Appl. 146 (1987) 65-96. 